ASSESSMENT OF SURFACE WATER BODIES USING AUTOMATED ALGORITHM, WEST GODAVARI DISTRICT, ANDHRA PRADESH, INDIA

\author{
KOLLI MEHAR GANESH ${ }^{1}$, RACHAKONDA SUBBA RAO ${ }^{2}$, Y.S.S.R.MURTHY ${ }^{3}$ \& P.A.R.K.RAJU ${ }^{4}$ \\ ${ }^{1,4}$ Professor Department of Civil Engineering, S. R. K. R. Engineering College, Andra Pradesh, India \\ ${ }^{2}$ Professor Department of Mathematics, S.R.K.R. Engineering College, Andra pradesh, India \\ ${ }^{3}$ Professor Department of Information Technology, S.R.K.R.Engineering College, Andra pradesh, India
}

\begin{abstract}
In the recent past lot of research is taking place on surface water bodies. The surface water includes lakes, ponds, rivers, streams and other exposed inland water bodies. There will be variation in spatial extent of these features as a function of rainfall amounts, the intensity of rainfall, etc. after season / year. Remote sensing, providing lot of data and extracting a lot of information over the changes from time to time. Nowadays the role of satellite image process is widely used in extraction of water bodies. Different researchers are using various methods to delineate water bodies from different satellite imagery varying in characteristics like spatial, spectral, and temporal. Our present study, includes an automatic approach to capture the water body, from a Resourcesat-2 AWiFS (Advanced Wide-Field Sensor) imagery, using an Automated Algorithm, for extraction of surface water body's model. The dynamics of surface water bodies in West Godavari district are studied through geospatial analysis for the extraction of water body layers for the month of December month 2016. Geospatial database on water body's information has been created from the Resourcesat-2 AWiFS image. The model was used for the estimation of the water spread area where bands 0.52-0.59 $\mu m$ (Green), 0.62-

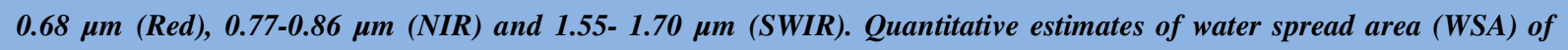
water bodies are obtained from analyzing inter / intra seasonal / annual analysis. The WSA calculated for each is 3782 ha. The information can be used in deciding the cropping pattern in the study area.

KEYWORDS: Surface Water Bodies, Water Spread Area, Remote Sensing \& Gis
\end{abstract}

Received: Sep 11, 2017; Accepted: Sep 30, 2017; Published: Oct 14, 2017; Paper Id.: IJCSEIERDOCT201710

\title{
INTRODUCTION
}

For the sustainability and integrity of the natural environment and in the social and economic development, one of the important factors is water. It is an indispensable tool for all the activities of the human being; it may be a groundwater, rainwater or recycled water. The day-to-day increase in demand, availability and cost factors are playing a major role in the utilization of the groundwater for water supply needs of townships and rural areas. The availability of good quality groundwater is more important than the quantity.

\section{Water Resources of India}

India experiences an average precipitation of 1,170 millimetres (46 in) per year, or about 4,000 cubic kilometres (960 cu mi) of rains annually or about 1,720 cubic metres $(61,000 \mathrm{cu} \mathrm{ft})$ of fresh water per person every year. Some 80 percent of its area experiences rains of 750 millimetres (30 in) or more a year. However, this rain is not uniform in time or geography. Most of the rains occur during its monsoon seasons (June to September), with 
the north east and north receiving far more rains than India's west and south. India is investing billions of amounts to harness the water resources for the sectors like irrigation industry and domestic consumption, which constitute about $15 \%$ of total public-sector outlay. India has connected nearly about 12 major river basins which are covering more than $250 \mathrm{M}$ ha with medium river basins and about 50 medium river basins which spread about $25 \mathrm{M}$ ha and other water bodies covering $7 \mathrm{M}$ ha with an irrigation potentiality near to $140 \mathrm{M}$ ha.

In the recent days extracting satellite imagery gain much importance. Mapping of forest areas, government assets, land use of crops and water bodies are some characteristics of importance in the satellite image. Both forest and water resources are subject to intensive exploitation and monitoring them at regular intervals is imperative for their sustainable management. One can observe the changes in the special features of water bodies over a season that it consists of different types of water bodies, i.e., rivers or streams, tanks, ponds and lakes. The disappearance and degradation of water bodies due to the global carbon cycle and climate variations are mapped into the spatiotemporal domain and are analysed to predict the amount of degradation. Space technology research centres are providing advanced tools to assess these variables which in turn help in taking the effective measures to improve the conditions.

The satellite data, observing changes in spatial, temporal and spectral characteristics are used by the various authors to generate thematic maps with special emphasis on water bodies and land use and land cover. Even though many authors developed different techniques to extract these features from satellite imagery, they have their own merits and demerits in extraction while working with high resolution data, visual interpretation of satellite data provides best delineation of water bodies of varied sizes, but it is time consuming.

In the stipulated time intervals satellite sensors can capture the existing situations of surface water features which facilitate to take the effective measures to improve the situation. It has also been observed that the satellite data utilization rapidly extended to other areas also.

The remote sensing data were downloaded from public domain (www.bhuvan.nrsc.gov.in) for free downloadable data on daily bases. This paper presents an approach to extract the water body from a Resourcesat-2 AWiFS imagery, using an Automated Algorithm, for extraction of surface water body model.

The typical spectral response of water facilitates its accurate identification and delineation on remotely sensed images. The time series data provide a record of changes in these storages. That's why, initiated a major effort in mapping surface water bodies at national level through development of automatic feature extraction techniques using multi-spectral data from multi-date IRS LISS III/AWiFS data sets. Satellite sensors can provide synoptic and dynamic coverage of the Earth's surface at frequent intervals which facilitates mapping, monitoring of dynamics of surface water bodies. Satellite data utilization for inventory, mapping and monitoring water bodies over larger areas is a popular technique for generation of dynamic databases. The present study is aimed at the automatic delineation of water bodies.

\section{MATERIALS AND METHODS}

The study area West Godavari district with its headquarters at 'Eluru' is one of the nine coastal districts of Andhra Pradesh state and is falling in the Survey of India toposheet no's 65 C, D, G \& H. It lies between the North Latitudes $16^{0}$ $51^{\prime}$ and $17^{0} 30^{\prime}$ and East Longitudes $80^{0} 50^{\prime}$ and $81^{0} 55^{\prime}$ and covers an area of about 7,795 sq.km; constituting $2.84 \%$ of 
the total area of the state and an average rainfall was $1078 \mathrm{~mm}$. The major profession of population in the district is the cultivation of Agricultural land. The density of the population is about 500 persons per sq.km, as per the central government information of 2011 census. The location map of the study area is shown in Figure 1.

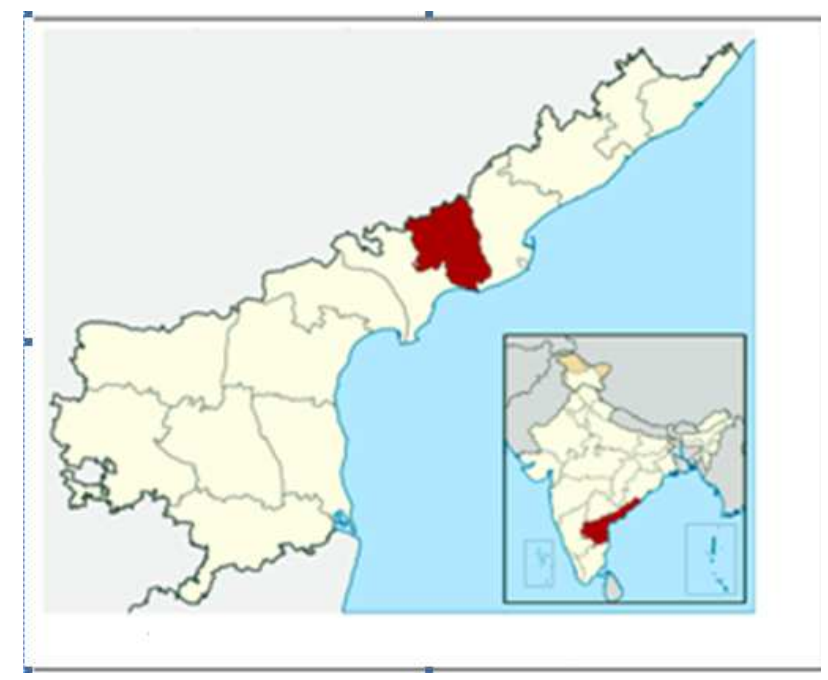

Figure 1: Location Map of the Study Area

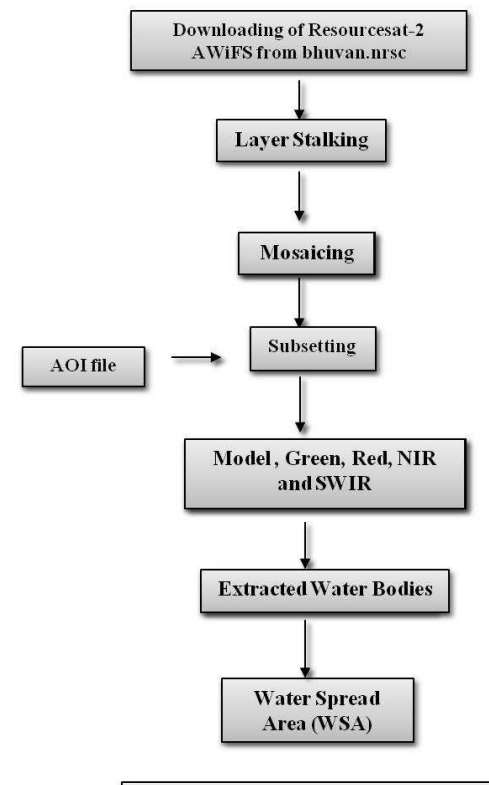

Figure 2: Overview of Methodology

Figure 2: Showing the Overall Methodology for the Extraction of the Water Bodies

\section{Data Used for Study}

The main source of satellite data for monitoring variations in water spread is through the missions of Resourcesat2, RISAT - 1 of Indian Remote Sensing (IRS). Various sensors are used in extracting the satellite data; they are listed in the table below.

\begin{tabular}{|c|c|c|}
\hline Satellite-Sensor & Spatial Resolution & Repetivity \\
\hline Resourcesat-2 AWiFS & $56 \mathrm{M}$ & 5 Days \\
\hline Resourcesat-2LiSS III & $23 \mathrm{~m}$ & 24 Days \\
\hline
\end{tabular}




\begin{tabular}{|c|c|c|}
\hline Year & Month & Source of Satellite Data \\
\hline 2016 & Dec, 2016 & Resourcesat -2 AWiFS \\
\hline
\end{tabular}

\section{Water Bodies Information System (WBIS)}

Water Bodies Information System (WBIS) is developed using web technologies like Vue JS, Leaflet and eCharts for data visualization and map elements. Raster data are automatically pooled from time-series water layers into a PostGIS enabled Postgres database. Java script and REST APIs are provided using node.js. And Cassandra for generating the statistics of an individual water body, regional (River Basin, River Sub-basin, State, District) Mapnik was used as rendering Engine. A process flowchart of WBIS is shown in Figure 3.

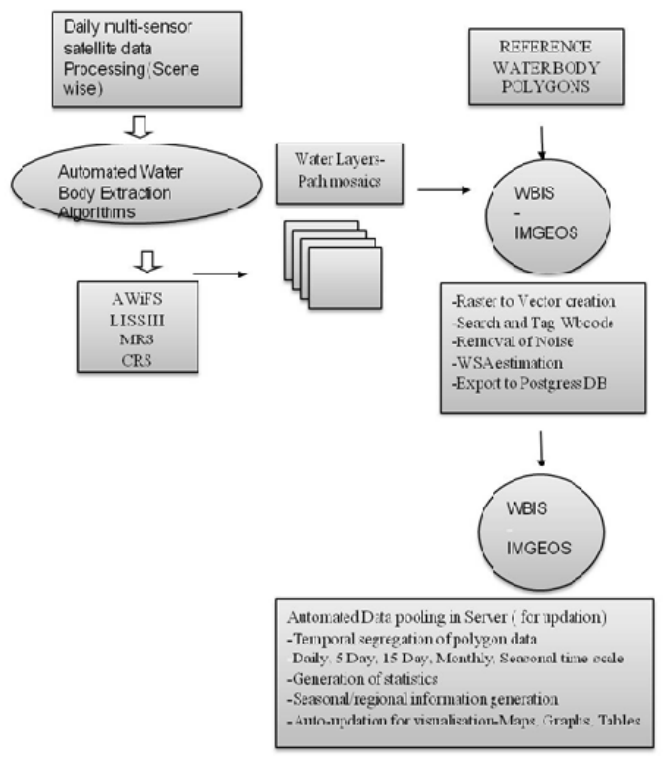

Figure 3: Process Flowchart of Water Bodies Information System

\section{RESULTS AND DISCUSSIONS}

This chapter describes the results obtained from the analysis of spatial-temporal satellite derived water body's information of West Godavari district, model framework for the extraction of surface water bodies from the Resourcesat-2 image of West Godavari district of Andhra Pradesh. The discussion is made from the satellite derived water body area with the help of figures. Water body layers derived from Resourcesat-2-AWiFS through the model for extraction of water body information for West Godavari district. The analysis is focused on the surface water bodies.

\section{Sub Setting of Study Area for Water Extraction}

The area of interest was subset by using a study area shape file to clip the required area by discussing in the methodology section.

\section{Extraction of Water Bodies from Satellite Image}

Extraction of water bodies, from the Resourcesat-2 image, for using the model is shown in methodology. The Figure 5 shows that, the extracted water body's layer, for the West Godavari district of Andhra Pradesh 


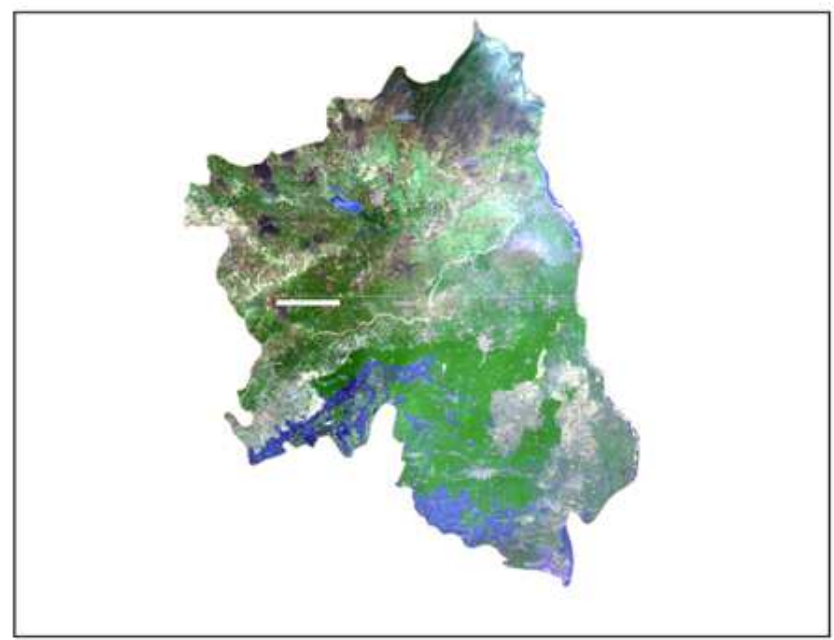

Figure 4: District Boundary Layer of Area of Interest

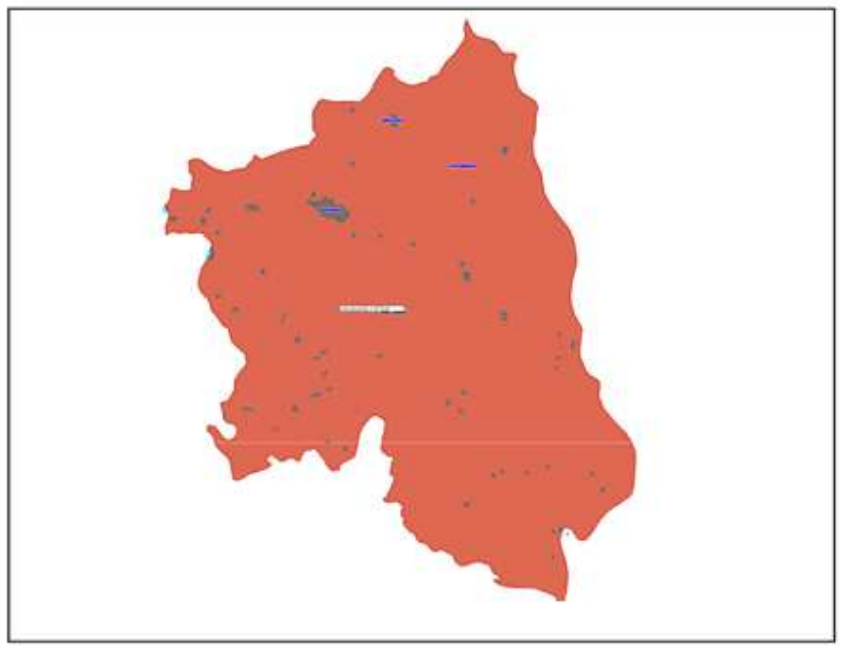

Figure 5: Extraction of Water Layer from Satellite Image

The above Figures 4 and 5 were shown that the water spread area of the individual water bodies. The total water spread area of the West Godavari by using the new model was 3782 ha. The model was extracted by pixel wise analysis has been done for the identification of water and non water bodies.

\section{CONCLUSIONS}

- The remote sensing and GIS application tools are very useful for the extraction of surface water body's information and analysis.

- The water spread area is analyzed in December month 2016. Satellite derived total WBA, number of water bodies categories by size as observed from satellite data and aggregated WBA in each category in the month of December-

- The total water spread area of West Godavari is 3782 ha.

- Total number of water bodies in West Godavari is 1741. 


\section{REFERENCES}

1. B. Lehner, and P.Doell, "Development and validation of a global databse of lakes, reservoirs and wetlands" J.of Hydrology, vol. 296, pp. 1-22, 2004.

2. Brochure, Central Groundwater board, 2013.

3. Hari $N \& N$ Bidyarani Chanu, Extraction of Surface Water Bodies from Landsat 8 of Prakasam district of Andhra Pradesh, Vol.7, Issue 3, Jun 2017, TJPRC Pvt., Ltd.

4. IPGS Design Document, NRSC-DPSD\&DBS-RS2-Aug2013.

5. IMGEOS Design document : NRSC-DPS-IMGEOS-Sept'10-TRI87.

6. Kshitij Mishra and P. Rama Chandra Prasad, Automatic Extraction of Water Bodies from Landsat Imagery Using Perceptron Model, Journal of Computational Environmental Sciences, Vol.2015, Article ID 903465.

7. McKee TB, Doeskin NJ, Kleist J (1993) The relationship of drought frequency and duration to time scales. In: Proceedings of the eighth conference on applied climatology, Anaheim, CA, January 17-23, 1993. American Meteorological Society Boston MA.179-184.

8. NRSC Document. Satellite derived Information on Water Bodies (WBA) and Water Bodies Fraction (WBF). NRSC-SDAPSARSAA-Feb 2014-TR-580.

9. NRSC Document. Near Real Time Extraction of Water bodies through Automated Algorithm from RISAT-1 MRS Datasets in IMGEOSNRSC - RSA /SDAPSA -Jun 2014.

10. Ouma, Y.O., and R. Tateishi, 2006. A water index for rapid mapping of shoreline changes of five East African Rift Valley lakes: An empirical analysis using Landsat TM and ETM data, International Journal of Remote Sensing, 27(15):3153-3181.

11. Praveen k Thakur., Velmurgan A., Aggarwal S P and Hariprasad.2011. Automatic extraction of information in glacial terrain using remote sensing. Journal of Hydrology.34: 65-75.

12. Dr.P.S.Roy, R.S.Dwivedi, D.Vijayan Remote Sensing \& GIS Applications,2010, NRSC, Hyderabad, 2010.

13. R.Ma,G.Yang,H.Duan,J.Jiang,S.Wang,X.Feng,A.Li,F.Kong,B.Xue,J.WuandS.Li, “China'slakesatpresent:number,areaandspatial distribution”,ScienceChinaEarthSciences, vol.54,pp.283-289,2011.

14. S. Subramaniam, A.V. Suresh Babu and P.S. Roy, "Automated Water Spread Mapping Using ResourceSat-1 AWiFS Data for Water Bodies Information System”. IEEE Journal of Selected Topics in Applied Earth Observations and Remote Sensing, vol.4, pp. $205-215,2011$.

15. Zhang Fang-fang., zhangBing., LI Jun-sheng., Qian, WuYuanfeng., and Song Yang. 2011. Comparative analysis of automatic water identification method based on Multispectral remote sensing. Procedia environmental scinces.11: 1482-1487. 